\title{
Subsidence of a metaphyseal-anchored press-fit stem after 4-year follow-up: an EBRA-FCA analysis
}

\author{
Dietmar Dammerer ${ }^{1} \cdot$ Philipp Blum $^{1}$ (C) David Putzer ${ }^{2} \cdot$ Dietmar Krappinger $^{1} \cdot$ Michael C. Liebensteiner $^{1}$. \\ Michael Nogler ${ }^{2} \cdot$ Martin Thaler $^{1}$
}

Received: 18 September 2020 / Accepted: 10 July 2021 / Published online: 21 July 2021

(c) The Author(s) 2021

\begin{abstract}
Purpose Uncemented stem migration analysis by EBRA-FCA (Einzel-Bild-Roentgen Analyse, Femoral Component Analyse) has been seen to be a good predictive indicator for early implant failure. In this study, we investigated the migration behavior of a cementless metaphyseal-anchored press-fit stem after 4-year follow-up.

Methods Applying a retrospective study design, we reviewed all consecutive patients who between 2012 and 2017 received a cementless Accolade II press-fit stem at our Department. We reviewed medical histories and performed radiological measurements using EBRA-FCA software. EBRA-FCA measurements and statistical investigations were performed by two independent investigators.

Results A total of 102 stems in 91 patients (female 60; male 31) fulfilled our inclusion criteria. Mean age at surgery was 66.2 (range 24.3-92.6) years. EBRA migration analysis showed a mean subsidence of $1.4 \mathrm{~mm}$ (range 0.0-12.0) at final follow-up. The angle between stem and femur axis was $0.5^{\circ}$ (range $0.0^{\circ}-2.8^{\circ}$ ) after 48 months. No correlations between gender or Dorr types and subsidence were found $(p>0.05)$. A body mass index $>30 \mathrm{~kg} / \mathrm{m}^{2}$ showed a significant increase in stem subsidence within the first $6(p=0.0258)$ and 12 months $(p=0.0466)$ postoperative.

Conclusions Migration pattern of the metaphyseal-anchored stem and a low subsidence rate at final follow-up may predict a good long-term clinical result.
\end{abstract}

Trial registration Number: 20181024-1875.

Keywords Stem subsidence $\cdot$ Total hip arthroplasty $\cdot$ Cementless $\cdot$ Einzel-Bild-Röntgen-Analyse (EBRA)

\section{Introduction}

Cemented as well as cementless femoral components in total hip arthroplasty (THA) yielded excellent long-term survival rates over $95 \%$ after 10 years [1]. Due to the literature, already 6 months after implantation of a primary cementless THA, aseptic loosening is described as the most common

Philipp Blum

philippblum1@gmx.de

Dietmar Dammerer

dietmar.dammerer@tirol-kliniken.at

David Putzer

david.putzer@i-med.ac.at

Dietmar Krappinger

dietmar.krappinger@tirol-kliniken.at

Michael C. Liebensteiner

michael.liebensteiner@i-med.ac.at cause of failure in THA [2-4]. Previously published studies reported distal migration of the femoral stem, called subsidence, which has shown to be a good predictive factor for early aseptic component loosening [5-8]. According to Krismer et al., distal migration of the stem of more than $1.5 \mathrm{~mm}$ $(\mathrm{mm})$ detected with Einzel-Bild-Roentgen Analyse-Femoral Component Analysis (EBRA-FCA) within the first 2 years

Michael Nogler

michael.nogler@i-med.ac.at

Martin Thaler

martin.thaler@i-med.ac.at

1 Department of Orthopaedics and Traumatology, Medical University of Innsbruck, Anichstrasse 35, 6020 Innsbruck, Austria

2 Department of Experimental Orthopaedics, Medical University of Innsbruck, Sonnenburgstr. 16, 6020 Innsbruck, Austria 
is a well-established risk factor for early implant failure [9]. However, comparability is limited due to the inclusion of cemented and cementless stems by Krismer et al. [9].

EBRA-FCA is a computer-assisted method for measuring the distal migration of femoral stems using standard anterior-posterior (ap) pelvic radiographs without requiring additional means at exposure (e.g. ball markers). It has proven accuracy and a sensitivity of more than $1 \mathrm{~mm}$ in detecting migration as compared to RSA (roentgen stereophotogrammetric analysis) [10,11].

The stem used in this study is the Accolade II by Stryker ${ }^{\circledR}$ (Stryker, Kalamazoo, MI, USA). It is designed for cementless, press-fit application and has a morphologic wedge with a size-specific medial curvature and a hydroxyapatite-coated proximal region [12]. As example given to the numerous arthroplasty registers around the world, in the Australian Orthopaedic Association National Joint Replacement Registry, 1961 Accolade II stems were implanted in 2018, which makes it the fifth most commonly used cementless stem in primary THA [13].

In the present study, we investigated the clinical results and the migration behavior of the cementless Accolade II stem using EBRA-FCA with a mid-term follow-up of up to 48 months. Furthermore, we evaluated the possible influence of gender, BMI and structural bone quality of the proximal femur on stem subsidence.

\section{Materials and methods}

The study was approved by the local ethics committee (Medical University of Innsbruck, Austria, Europe). We applied a retrospective study design and reviewed all consecutive patients who received an Accolade II stem at our department between 2012 and 2017. During this time, a total of 219 Accolade II stems were implanted as part of a primary THA.

We investigated the medical histories for sociodemographic data, surgical approach, body mass index, cutto-suture time and the preoperative diagnosis for THA indication. Furthermore, range of motion was recorded preoperatively and up to 1 year after surgery by surgeons at our department using a goniometer during clinical examination.

Axial stem migration, prosthetic stability as well as tilting of the stem were assessed retrospectively with EBRA-FCA from plain X-rays [9, 10]. A total of 19 reference points are defined on the femoral head $(n=7)$, the stem $(n=2)$, the femoral cortex $(n=8)$, and 1 each at the major and minor trochanter [10]. The EBRA-FCA software excludes radiographs with a comparability algorithm, which identifies significant positioning artifacts by comparing specific bone and prosthetic landmarks. Figure 1 shows the X-ray of an Accolade II stem including EBRA-FCA references.

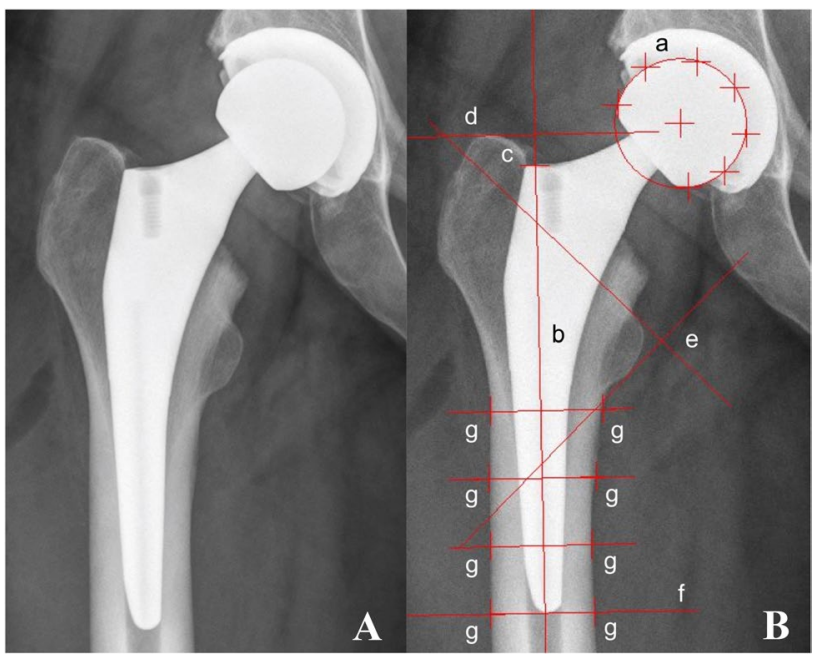

Fig. 1 Anterior to posterior X-rays showing an Accolade II stem (A) and with EBRA-FCA references (B) a head points b stem axis c stem shoulder d major trochanter line e minor trochanter lines f tip-of-stem line $\mathrm{g}$ points at femoral bone contour

In our department, we routinely follow patients with radiographs before discharge, 6 weeks after surgery, 12 months postoperative and then in a 1-2 year interval. We perform additional radiographs if the patient has any complaints with the THA. All radiographs were taken at our Department of Radiology with the same technique [anterior-posterior (AP) radiographs; patient standing in upright position and full weight bearing]. For our EBRA investigation, a minimum of four radiographs per patient and a minimum radiological follow-up of up to 6 months was required for this analysis. Migration analysis was done with EBRA by one independent investigator, who was not involved in the surgeries or postoperative treatment of patients.

Our postoperative protocol allows immediate full weight bearing depending on pain after cementless primary THA from day one after surgery.

\section{Statistics}

Mean, median, range, and standard deviation were calculated for the various measurement parameters. For the analysis, Access and Excel (Microsoft Office Professional Plus 2010, Redmond, WA, USA) as well as Graph Pad Prism (Version 8.0, GraphPad Software, Inc., La Jolla, CA, USA) were used. All data were tested for normality using the Kolmogorov-Smirnov test. For comparison of the EBRA-FCA measurements at different time steps as well as for comparison of subsidence according to the Dorr classification the Kruskal-Wallis test was used. The EBRA measurements were compared by BMI and gender classification using the Mann-Whitney $U$ test. When comparing the range of motion pre- and postoperatively, the Mann-Whitney $U$ test was used 
for comparison. A $p$ value of 0.05 was considered statistically significant.

\section{Results}

A total of 102 stems in 91 patients (female 60; male 31) fulfilled our inclusion criteria. In 11 patients, the Accolade II stem was implanted bilaterally. Mean patient age at surgery was 66.2 (range 24.3-92.6) years and mean body mass index was 27.2 (range 16.2-40.1) kg/m². Mean follow-up was 48 (range 10-65) months. The preoperative diagnosis was osteoarthritis in 94 (92.2\%) hips, avascular necrosis of the femoral head in 7 (6.8\%) hips and a pathologic fracture in $1(1.0 \%)$ hip. The mean cut-to-suture time was 63 (range 29-161) min. The investigated stem was combined with cementless press-fit cups. All surgeries were performed in a supine position using the direct anterior approach [14]. The most used head size was $32 \mathrm{~mm}(62.7 \%)$. More details are shown in Tables 1 and 2.

EBRA-FCA analysis at 48 months follow-up was calculated for 50 out of 102 stems with an EBRA-FCA-given comparability limit of $3.0 \mathrm{~mm}$ ( $95 \%$ confidence interval). A total of $637 \mathrm{x}$ rays were analyzed, whereby 44 radiographs (6.9\%) were rejected by the EBRA-FCA software. On average, 6.2 (range 4-20) x rays per implant were analyzed. None of our patients had to be excluded from EBRA-FCA migration analysis. A complete set of radiographs at every single time step (e.g. 6 months, 12 months, etc.) was not

Table 1 Patient demographics for the study group

\begin{tabular}{ll}
\hline Number of patients & 60 \\
Female & 31 \\
Male & 91 \\
Total & $66.2(24.3-92.6)$ \\
Mean age (years) & $27.2(16.2-40.1)$ \\
BMI (kg/m $\left.{ }^{2}\right)$ & $63(29-161)$ \\
Cut-to-suture time (min) & \\
Surgical approach & 102 \\
Direct anterior approach & \\
Surgical position & 102 \\
Supine & \\
Preoperative diagnosis & 94 \\
Osteoarthritis & 7 \\
Avascular necrosis of the femoral head & 1 \\
Pathologic fracture & \\
Dorr classification & 14 \\
Type A & 83 \\
Type B & 5 \\
Type C & \\
\hline
\end{tabular}

Range is given in brackets
Table 2 Details of implanted components

\begin{tabular}{ll}
\hline Stem product & \\
Stryker Accolade II & $102[100.0]$ \\
CCD angle & \\
$127^{\circ}$ & $80[78.4]$ \\
$132^{\circ}$ & $22[21.6]$ \\
Head size $(\mathrm{mm})$ & \\
22 & $1[1.0]$ \\
26 & $1[1.0]$ \\
28 & $30[29.4]$ \\
32 & $64[62.7]$ \\
36 & $6[5.9]$ \\
Cup product & \\
Stryker Trident PSL & $101[99.0]$ \\
DePuy Bantam & $1[1.0]$ \\
\hline
\end{tabular}

Percentages are given in brackets

available for each stem in our study. Therefore, total subsidence could not be calculated in all cases. This gives a different number of cases in the corresponding migration behavior analysis over time.

The EBRA-FCA analysis showed a mean migration of $0.7 \mathrm{~mm}$ (range $0.0-8.5$ ) at 6 months, $1.2 \mathrm{~mm}$ (range $0.0-12.0$ ) at 12 months, $1.4 \mathrm{~mm}$ (range $0.0-11.0$ ) at 24 months, $1.4 \mathrm{~mm}$ (range 0.1-6.6) at 36 months and $1.4 \mathrm{~mm}$ (range $0.1-5.5$ ) at 48 months after surgery. Thus, the main axial subsidence occurred particularly in the first postoperative year (Table 3; Fig. 2). The calculated mean axial implant migration was $0.11 \mathrm{~mm}$ in the first 6 months, $0.10 \mathrm{~mm}$ between 6 and 12 months, $0.02 \mathrm{~mm}$ between 12 and 24 months and $0.00 \mathrm{~mm}$ thereafter.

In addition, the angle between stem and femur axis was $0.2^{\circ}$ (range $0.0^{\circ}-0.9^{\circ}$ ) after 6 months, $0.3^{\circ}$ (range $0.0^{\circ}-1.5^{\circ}$ ) after 12 months, $0.4^{\circ}$ (range $0.0^{\circ}-1.7^{\circ}$ ) after 24 months, $0.5^{\circ}$ (range $0.0^{\circ}-2.6^{\circ}$ ) after 36 months and $0.5^{\circ}$ (range $0.0^{\circ}-2.8^{\circ}$ ) after 48 months (Fig. 3).

A statistically significant smaller angle was found at 6 months in comparison to 12 months $(p=0.0191)$, 24 months $(p=0.0011), 36$ months $(p<0.0001)$ and 48 months $(p<0.0001)$. Also, statistically significant less initial subsidence was found at 6 months in comparison to 12 months $(p=0.0235), 24$ months $(p<0.0001), 36$ months $(p<0.0001)$ and 48 months $(p<0.0001)$.

Of 103 stems 64 had sufficient EBRA-FCA follow-up to assess migration behavior after 2 years. Working from the critical threshold values, 18 (28.2\%) of 65 stems showed an axial subsidence of more than $1.5 \mathrm{~mm}$. An axial subsidence of more than $2.7 \mathrm{~mm}$ was detected in nine (14.1\%) of 64 cases 2 years postoperatively. Percentages of migrated stems are given in Table 4. The most severe stem subsidence $(12.0 \mathrm{~mm})$ was observed in a patient within the first 
Table 3 Mean total subsidence in millimeters $(\mathrm{mm})$ over time

\begin{tabular}{llllll}
\hline & 6 months $(n=100)$ & 12 months $(n=39)$ & 24 months $(n=64)$ & 36 months $(n=66)$ & 48 months $(n=50)$ \\
\hline $\begin{array}{c}\text { Subsidence of the Accolade II stem in } \\
\text { mm (range) }\end{array}$ & $0.7(0.0-8.5)$ & $1.2(0.0-12.0)$ & $1.4(0.0-11.0)$ & $1.4(0.1-6.6)$ & $1.4(0.1-5.5)$ \\
\hline
\end{tabular}

Range is given in brackets

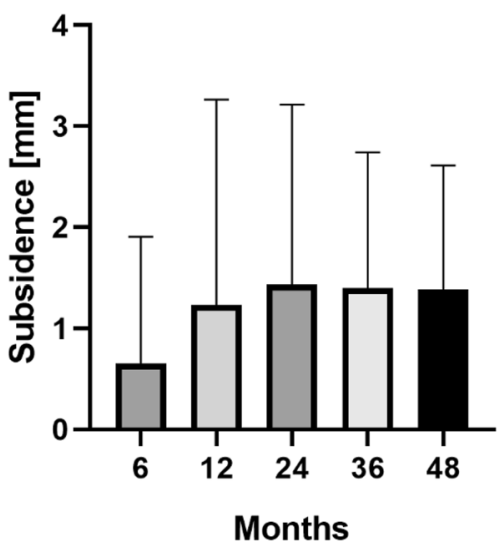

Fig. 2 Mean and standard deviation (bars) of total stem subsidence for the clinical follow-up of 48 months

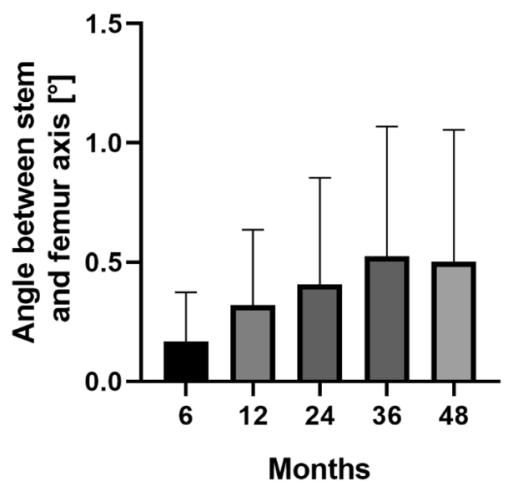

Fig. 3 Mean and standard deviation (bars) of the angle between stem and femur axis for the clinical follow-up of 48 months
12 months. Postoperative X-rays of this patient were reevaluated and undersizing of the stem was identified as probable cause for subsidence. However, no postoperative complications were observed in this case, e.g. stem revision, limping or pain. The procedure for this patient is shown in Fig. 4. Additionally, another implant showed a subsidence of $11 \mathrm{~mm}$ after 24 months. In this case, undersizing was ruled out and no postoperative complications (e.g. stem revision, etc.) were documented.

According to the Dorr classification, we divided our patient cohort into three groups to measure its effect on subsidence [15]: patients with femoral bone classification Type A, Type B and Type C. There was no statistically significant difference in the total migration between the three sub-cohorts $(p>0.05)$. Nevertheless, the stem subsidence in Type $\mathrm{C}$ femurs was higher than the overall mean subsidence at last measurement in 3/5 (60\%) stems. A statistically larger angle was found at 6 months for Type A in comparison to Type $\mathrm{C}$ femuras $(p=0.0265)$. Additionally, the patients were divided into groups according to their BMI: normal $\left(\mathrm{BMI} \leq 25 \mathrm{~kg} / \mathrm{m}^{2}\right.$ ), overweight (BMI $25.1-29.9 \mathrm{~kg} / \mathrm{m}^{2}$ ) and obese (BMI $\left.\geq 30 \mathrm{~kg} / \mathrm{m}^{2}\right)$. A statistically significant greater subsidence of the stem was found for obese patients within 6 months $(p=0.0258)$ and within 12 months $(p=0.0466)$ of surgery in comparison to normal and overweight patients. No statistically significant difference was found between obese and normal to overweight patients when considering the variation in the stem angle. Furthermore, EBRA measurements for stem angle variations and subsidence between female and male patients showed no statistically significant difference $(p>0.05)$.

Table 4 Total subsidence in millimeters $(\mathrm{mm})$ over time

\begin{tabular}{|c|c|c|c|c|c|}
\hline $\begin{array}{l}\text { Total subsidence } \\
(\mathrm{mm})\end{array}$ & 6 months $(n=100)$ & 12 months $(n=39)$ & 24 months $(n=64)$ & 36 months $(n=66)$ & 48 months $(n=50)$ \\
\hline$\leq 1.5$ & $89(89.0)$ & $30(76.9)$ & $46(71.8)$ & $44(66.7)$ & $32(64.0)$ \\
\hline$>1.5$ & $6(6.0)$ & $5(12.8)$ & $9(14.1)$ & $13(19.7)$ & $12(24.0)$ \\
\hline$>2.7$ & $5(5.0)$ & $4(10.3)$ & $9(14.1)$ & $9(13.6)$ & $6(12.0)$ \\
\hline
\end{tabular}

Percentages are given in brackets 


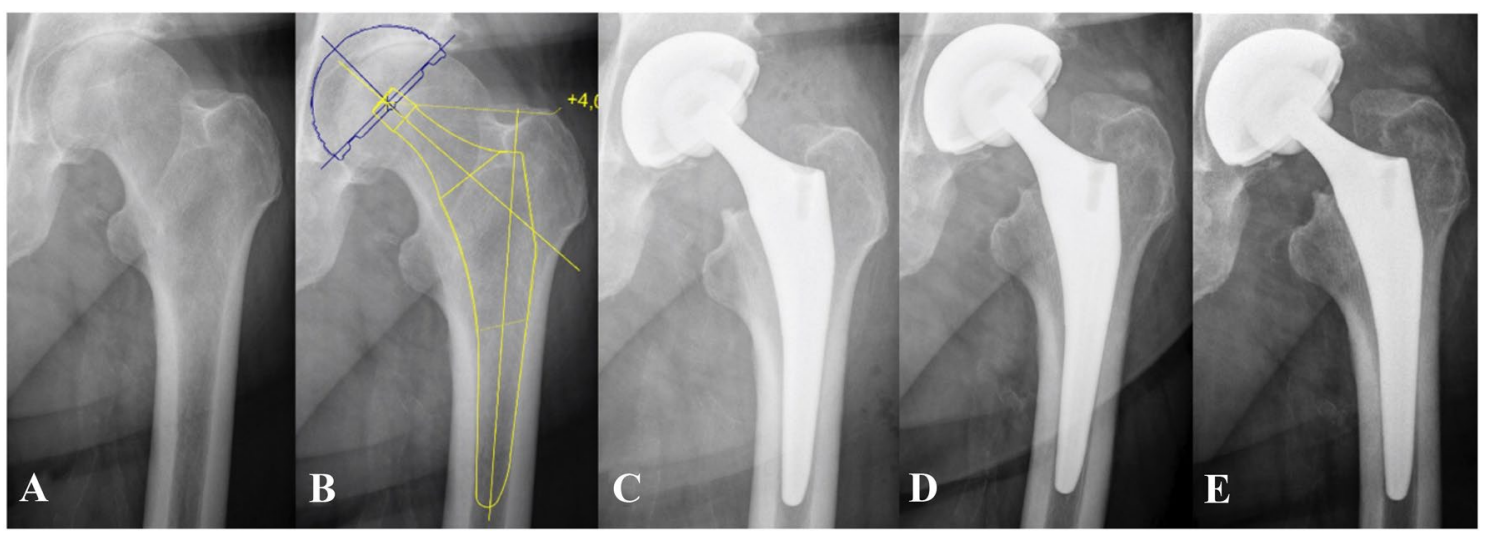

Fig. 4 The X-ray series presents the procedure of the patient with the largest detected subsidence A preoperative situation showing osteoarthritis of the hip $\mathbf{B}$ preoperative prosthetic planning $\mathbf{C}$ immediate

Pre- and postoperative comparison of the range of motion showed a mean improvement in flexion of $10^{\circ}$ (range $-45^{\circ}$ to $40^{\circ}, p<0.0001$ ), internal rotation $13^{\circ}$ (range $-20^{\circ}$ to $40^{\circ}, p<0.0001$ ), external rotation $8^{\circ}$ (range $-20^{\circ}$ to $40^{\circ}$, $p<0.0001$ ), abduction $13^{\circ}$ (range $-30^{\circ}$ to $45^{\circ}, p<0.0001$ ) and adduction $11^{\circ}$ (range $-20^{\circ}$ to $30^{\circ}, p<0.0001$ ). Preoperatively, flexion $\geq 90^{\circ}$ was possible in $69.9 \%$ of the investigated hip joints; this figure increased to $93.2 \%$ postoperatively.

In addition, postoperative complications were documented. In four cases $(3.9 \%)$, a fracture occurred after implantation. In another five cases $(4.9 \%)$, a revision procedure had to be performed. Of these, four cases showed migration $>1.5 \mathrm{~mm}$ and thereof three cases $>2.7 \mathrm{~mm}$ within the first 2 years. Two cases of revision were carried out in Dorr Type $\mathrm{C}$ femurs. No infection was observed overall.

\section{Discussion}

As THA is one of the most successful medical procedures, various stem designs are available. Short stems have been clinically established for years with significant differences between femoral fixation concepts [16]. The present study analyzed the migration behavior of the cementless methaphyseal-anchored Accolade II stem. To the best of our knowledge, this is the first study to investigate the mean subsidence of the Accolade II stem using EBRA-FCA. After a follow-up of 48 months, we can report a low mean subsidence of $1.4 \mathrm{~mm}$ and a small axial deviation of $0.5^{\circ}$ between stem and femur axis. In addition, more than $85 \%$ of the stems showed a subsidence of less than $2.7 \mathrm{~mm}$ and, although more than $70 \%$, less than $1.5 \mathrm{~mm}$ after 24 months. Therefore, our results are well in line with previously published postoperative X-ray D subsidence of $5.3 \mathrm{~mm}$ was detected by EBRAFCA 6 weeks after surgery $\mathbf{E}$ subsidence of $12 \mathrm{~mm}$ was detected by EBRA-FCA 12 months after surgery

EBRA-FCA studies, and thus good long-term implant survival of the investigated femoral stem may be expected.

Primary stability is a prerequisite for the bony ingrowth of the implant in THA. Working from this assumption, increased subsidence of the femoral component within the first 2 years after surgery was described as a risk factor for later aseptic loosening [9]. With a specificity of $100 \%$ and a sensitivity of $78 \%$ for detection of migration of more than $1 \mathrm{~mm}$, as compared with roentgen stereophotogrammetric analysis (RSA), EBRA-FCA is suitable for identifying and measuring the subsidence of femoral components in THA [10]. While RSA is considered to be the gold standard for migration measurement, EBRA-FCA offers the advantage of being a non-invasive method that can be used in our retrospective study design.

Different thresholds for prediction of aseptic loosening have been described in the given literature [5, 9]. In our study, $28.2 \%$ of the stems showed a migration of more than $1.5 \mathrm{~mm}$ and $14.1 \%$ of more than $2.7 \mathrm{~mm} 2$ years postoperatively. Similar results were recently published by Kutzner et al. for the calcar-guided Optimys stem (Mathys Ltd Bettlach, Bettlach, Switzerland), stating that $38.2 \%$ and $15.2 \%$, respectively, showed a subsidence of more than $1.5 \mathrm{~mm}$ and $2.7 \mathrm{~mm}$ after 2 years [17]. Tian et al. also investigated the Accolade II stem, with only one of 1017 (0.1\%) implants subsiding more than $1.5 \mathrm{~mm}$ after 2 years [18]. While in previous mentioned studies, EBRA-FCA was used to determine subsidence, the measurement method used by Tian et al. cannot be exactly followed from their study protocol. The different measurement methods might be a reason for the large differences in detected subsidence after 2 years.

Migration analysis of various metaphyseal-anchored femoral short stems has already been part of several shortto mid-term EBRA studies (15-20) [17, 19-23]. Kutzner et al. showed a mean subsidence of $1.39 \mathrm{~mm}$ after 2 years 
and $1.5 \mathrm{~mm}$ after 5 years for the Optimys stem [17]. When investigating the same stem, Schaer et al. reported a slightly larger mean axial migration of $1.71 \mathrm{~mm}$ and $2.04 \mathrm{~mm}$ after 2 and 5 years, respectively [22]. While Schmidutz et al. found a mean subsidence of $0.7 \mathrm{~mm}$ after 2.7 years for the Metha stem (B. Braun Aesculap, Tuttlingen, Germany), a mean subsidence of $1.01 \mathrm{~mm} 2$ and 3 years after surgery was reported by Jahnke et al. for the same stem [20, 23]. In a study by Freitag et al., the Fitmore stem (Zimmer Biomet, Warsaw, IN, USA) showed $1.1 \mathrm{~mm}$ of axial migration after 2 and 5 years [21]. As compared to these results, mean subsidence in our study was $1.4 \mathrm{~mm}$ after 2 and 4 years. Nearly all the above-mentioned implants showed initial subsidence with secondary stabilization after 2 years. This phenomenon, already described by Krismer et al. in 1999, is not uncommon and can lead to long-lasting survival of the implant [9]. Main subsidence of the Accolade II stem was observed during the first postoperative year. Mean monthly migration reduced from $0.11 \mathrm{~mm}$ during the first 6 months after surgery to less than $0.02 \mathrm{~mm}$ after 24 months. As a result, a stable implant situation between 1 and 2 years postoperatively can be assumed.

Several factors potentially affecting the subsidence of femoral components have already been investigated. The influence of patient demographics like weight, BMI and gender on stem subsidence yielded different results. Stihsen et al. observed a significant impact of body weight over $75 \mathrm{~kg}$ on subsidence of the Vision 2000 stem (Depuy, Warsaw, IN, USA) [24]. These findings were confirmed by Kutzner et al. for the Optimys stem [25]. In contrast to body weight, a BMI $>30 \mathrm{~kg} / \mathrm{m}^{2}$ had no influence on subsidence in previous studies [24, 25]. However, in a study by Freitag et al., the Fitmore stem showed a tendency to greater subsidence in patients with a BMI $>30 \mathrm{~kg} / \mathrm{m}^{2}$ without finding significant differences [26]. In our study, we demonstrate a statistically significant greater subsidence of the Accolade II stem in obese patients within 6 months $(p=0.0258)$ and 12 months ( $p=0.0466)$ of follow-up in comparison to normal and overweight patients. While some studies showed male gender to be at risk for greater subsidence [17, 24], we cannot report a statistically significant difference between female and male patients.

In addition to patient demographics, press-fit of the stem is considered to be one of the key factors [27]. A connection between femoral configuration and axial migration was detected by Jahnke et al. for the Metha short stem [28]. In our study, the preoperative Dorr types of femoral configuration showed no significant influence on subsidence of the Accolade II stem, whereby stem subsidence in Type $\mathrm{C}$ femurs was higher than mean subsidence in $60 \%$. The subsidence of the Optimys stem was also not influenced by the Dorr types, but Schaer et al. found a correlation between Optimys stem size and subsidence, with stem size $\geq 6$ showing a larger subsidence than stem size $<6$ at 5-year follow-up [22]. However, this phenomenon was observed only in women. Schaer et al. concluded that the surgeon may not have chosen an even larger implant out of fear of an intraoperative periprosthetic fracture, as women tend to require smaller implants than men [22].

This study has several limitations, including the absence of a control group and retrospective methodology. As a result, some of the treated patients had to be excluded from the cohort, possibly making the study more prone to selection bias. Nevertheless, our number of cases is larger than in some of the cited studies [20-23]. Therefore, valid results can be assumed. Furthermore, the number of radiographs and duration of follow-up varied for each hip. This may have influenced the migration results due to the smoothing function in the software and made it difficult to follow the exact outcome of each individual implant. Due to the retrospective character of this study, we could not rule out all factors influencing stem subsidence. As already known, stem subsidence is multifactorial influenced by e.g. BMI, undersizing the stem etc.; all these factors may be a contributor for early migration. Especially worth mentioning is undersizing, a well-known obstacle and problem by using a metaphyseal-anchored press-fit stem in THA. This problem could not be ruled out in all cases because of the retrospective study character. To undersize a femoral stem is and will be a common problem in THA, nevertheless in some cases, the stem will stay in place over years and in some other cases, the stem will migrate very early. $\mathrm{We}$, therefore, advice the surgeon to be aware of this problem and should have a more frequently follow-up of these patients. In addition, the number of Dorr $\mathrm{C}$ type femurs may have been too small to detect significant differences and a power analysis was not performed.

In summary, the EBRA-FCA analysis for the cementless Accolade II short stem showed a small mean subsidence and low mean tilting of the stem axis with good clinical function up to 4 years after surgery. After an initial subsidence in the first postoperative year, the mean monthly migration rate reduced and a stable implant position may be subsequently predicted. While gender and Dorr classification had no influence on subsidence, a statistically significant greater subsidence of the stem was found for obese patients within 6 months and 12 months. Further observations are necessary to confirm the expected good long-term results.

Acknowledgements This research did not receive any specific grant from funding agencies in the public, commercial, or not-for-profit sectors.

Author contributions All listed authors have substantially contributed to this study. All authors have reviewed and confirmed the accuracy of the whole manuscript. 
Funding Open access funding provided by University of Innsbruck and Medical University of Innsbruck. This research did not receive any specific grant from funding agencies in the public, commercial, or not-for-profit sectors.

Data availability Data will be sent if necessary.

Code availability Not applicable.

\section{Declarations}

Conflict of interest The authors Martin Thaler and Michael Nogler have received research grants from Stryker that are not related to the content of the study. Martin Thaler is Treasurer of the European Hip Society. Dietmar Dammerer, Philipp Blum, David Putzer, Dietmar Krappinger and Michael C. Liebensteiner declare that they have no conflicts of interests.

Ethical approval The local ethics committee approved the study.

Consent to participate Not applicable. Retrospective study design.

Consent for publication All authors have reviewed and confirmed the accuracy of the whole manuscript.

Open Access This article is licensed under a Creative Commons Attribution 4.0 International License, which permits use, sharing, adaptation, distribution and reproduction in any medium or format, as long as you give appropriate credit to the original author(s) and the source, provide a link to the Creative Commons licence, and indicate if changes were made. The images or other third party material in this article are included in the article's Creative Commons licence, unless indicated otherwise in a credit line to the material. If material is not included in the article's Creative Commons licence and your intended use is not permitted by statutory regulation or exceeds the permitted use, you will need to obtain permission directly from the copyright holder. To view a copy of this licence, visit http://creativecommons.org/licenses/by/4.0/.

\section{References}

1. Kärrholm J, Mohaddes M, Odin D, Vinblad J, Rogmark C, Rolfson O (2018) Swedish hip arthroplasty register annual report 2017. Swedish Hip Arthroplasty Register

2. Johnsen SP, Sørensen HT, Lucht U, Søballe K, Overgaard S, Pedersen AB (2006) Patient-related predictors of implant failure after primary total hip replacement in the initial, short- and long-terms. A nationwide Danish follow-up study including 36,984 patients. J Bone Joint Surg Br 88:1303-1308. https://doi.org/10.1302/0301620X.88B10.17399

3. Ulrich SD, Seyler TM, Bennett D, Delanois RE, Saleh KJ, Thongtrangan I, Kuskowski M, Cheng EY, Sharkey PF, Parvizi J, Stiehl JB, Mont MA (2008) Total hip arthroplasties: what are the reasons for revision? Int Orthop 32:597-604. https://doi.org/10. 1007/s00264-007-0364-3

4. Sadoghi P, Liebensteiner M, Agreiter M, Leithner A, Böhler N, Labek G (2013) Revision surgery after total joint arthroplasty: a complication-based analysis using worldwide arthroplasty registers. J Arthroplasty 28:1329-1332. https://doi.org/10.1016/j.arth. 2013.01.012

5. Streit MR, Haeussler D, Bruckner T, Proctor T, Innmann MM, Merle C, Gotterbarm T, Weiss S (2016) Early migration predicts aseptic loosening of cementless femoral stems: a long-term study. Clin Orthop Relat Res 474:1697-1706. https://doi.org/10.1007/ s11999-016-4857-5

6. Kroell A, Beaulé P, Krismer M, Behensky H, Stoeckl B, Biedermann R (2009) Aseptic stem loosening in primary THA: migration analysis of cemented and cementless fixation. Int Orthop 33:1501-1505. https://doi.org/10.1007/s00264-008-0701-1

7. Walker PS, Mai SF, Cobb AG, Bentley G, Hua J (1995) Prediction of clinical outcome of THR from migration measurements on standard radiographs. A study of cemented Charnley and Stanmore femoral stems. J Bone Joint Surg Br 77:705-714

8. Freeman MA, Plante-Bordeneuve P (1994) Early migration and late aseptic failure of proximal femoral prostheses. J Bone Joint Surg Br 76:432-438

9. Krismer M, Biedermann R, Stöckl B, Fischer M, Bauer R, Haid C (1999) The prediction of failure of the stem in THR by measurement of early migration using EBRA-FCA. Einzel-Bild-Roentgen-Analyse-femoral component analysis. J Bone Joint Surg Br 81:273-280. https://doi.org/10.1302/0301-620x.81b2.8840

10. Biedermann R, Krismer M, Stöckl B, Mayrhofer P, Ornstein E, Franzén H (1999) Accuracy of EBRA-FCA in the measurement of migration of femoral components of total hip replacement. EinzelBild-Röntgen-Analyse-femoral component analysis. J Bone Joint Surg Br 81:266-272. https://doi.org/10.1302/0301-620x.81b2. 8842

11. Abrahams JM, Callary SA, Jang SW, Hewitt J, Howie DW, Solomon LB (2020) Accuracy of EBRA-cup measurements after reconstruction of severe acetabular defects at revision THR. J Orthop Res. https://doi.org/10.1002/jor.24623

12. Stryker (2012) Accolade II femoral hip system. https://www.stryk ermeded.com/media/1210/accolade-ii-surgical-technique.pdf. Accessed 10 Sept 2020

13. AOANJR Registry (2019) Annual report 2019. https://aoanjrr. sahmri.com/documents/10180/668596/Hip\%2C+Knee+\%26+ Shoulder+Arthroplasty/c287d2a3-22df-a3bb-37a2-91e6c00bfc f0. Accessed 10 Sept 2020

14. Meermans G, Konan S, Das R, Volpin A, Haddad FS (2017) The direct anterior approach in total hip arthroplasty: a systematic review of the literature. Bone Joint J 99-B:732-740. https://doi. org/10.1302/0301-620X.99B6.38053

15. Dorr LD, Faugere MC, Mackel AM, Gruen TA, Bognar B, Malluche HH (1993) Structural and cellular assessment of bone quality of proximal femur. Bone 14:231-242. https://doi.org/10.1016/ 8756-3282(93)90146-2

16. Falez F, Casella F, Papalia M (2015) Current concepts, classification, and results in short stem hip arthroplasty. Orthopedics 38:S6-13. https://doi.org/10.3928/01477447-20150215-50

17. Kutzner KP, Ried E, Donner S, Bieger R, Pfeil J, Freitag T (2020) Mid-term migration pattern of a calcar-guided short stem: a fiveyear EBRA-FCA-study. J Orthop Sci. https://doi.org/10.1016/j. jos.2020.01.001

18. Tian S, Goswami K, Manrique J, Blevins K, Azboy I, Hozack WJ (2019) Direct anterior approach total hip arthroplasty using a morphometrically optimized femoral stem, a conventional operating table, without fluoroscopy. J Arthroplasty 34:327-332. https:// doi.org/10.1016/j.arth.2018.10.023

19. White CA, Carsen S, Rasuli K, Feibel RJ, Kim PR, Beaulé PE (2012) High incidence of migration with poor initial fixation of the Accolade stem. Clin Orthop Relat Res 470:410-417. https:// doi.org/10.1007/s11999-011-2160-Z

20. Schmidutz F, Graf T, Mazoochian F, Fottner A, Bauer-Melnyk A, Jansson V (2012) Migration analysis of a metaphyseal anchored short-stem hip prosthesis. Acta Orthop 83:360-365. https://doi. org/10.3109/17453674.2012.712891

21. Freitag T, Fuchs M, Woelfle-Roos JV, Reichel H, Bieger R (2019) Mid-term migration analysis of a femoral short-stem prosthesis: a 
five-year EBRA-FCA-study. Hip Int 29:128-133. https://doi.org/ $10.1177 / 1120700018772277$

22. Schaer MO, Finsterwald M, Holweg I, Dimitriou D, Antoniadis A, Helmy N (2019) Migration analysis of a metaphyseal-anchored short femoral stem in cementless THA and factors affecting the stem subsidence. BMC Musculoskelet Disord 20:604. https://doi. org/10.1186/s12891-019-2980-7

23. Jahnke A, Wiesmair AK, Ulloa CAF, Ahmed GA, Rickert M, Ishaque BA (2020) Outcome of short- to medium-term migration analysis of a cementless short stem total hip arthroplasty using EBRA-FCA: a radiological and clinical study. Arch Orthop Trauma Surg 140:247-253. https://doi.org/10.1007/ s00402-019-03315-3

24. Stihsen C, Radl R, Keshmiri A, Rehak P, Windhager R (2012) Subsidence of a cementless femoral component influenced by body weight and body mass index. Int Orthop 36:941-947. https:// doi.org/10.1007/s00264-011-1360-1

25. Kutzner KP, Kovacevic MP, Freitag T, Fuchs A, Reichel H, Bieger R (2016) Influence of patient-related characteristics on early migration in calcar-guided short-stem total hip arthroplasty: a 2-year migration analysis using EBRA-FCA. J Orthop Surg Res 11:29. https://doi.org/10.1186/s13018-016-0363-4

26. Freitag T, Kappe T, Fuchs M, Jung S, Reichel H, Bieger R (2014) Migration pattern of a femoral short-stem prosthesis: a 2-year EBRA-FCA-study. Arch Orthop Trauma Surg 134:1003-1008. https://doi.org/10.1007/s00402-014-1984-x

27. Viceconti M, Brusi G, Pancanti A, Cristofolini L (2006) Primary stability of an anatomical cementless hip stem: a statistical analysis. J Biomech 39:1169-1179. https://doi.org/10.1016/j.jbiomech. 2005.03.024

28. Jahnke A, Engl S, Seeger JB, Basad E, Rickert M, Ishaque BA (2015) Influences of fit and fill following hip arthroplasty using a cementless short-stem prosthesis. Arch Orthop Trauma Surg 135:1609-1614. https://doi.org/10.1007/s00402-015-2302-y

Publisher's Note Springer Nature remains neutral with regard to jurisdictional claims in published maps and institutional affiliations. 